\title{
ZoomNet: Part-Aware Adaptive Zooming Neural Network for 3D Object Detection
}

\author{
Zhenbo Xu, ${ }^{1}$ Wei Zhang, ${ }^{2}$ Xiaoqing Ye, ${ }^{2}$ Xiao Tan, ${ }^{2}$ Wei Yang, ${ }^{*}$ Shilei Wen, ${ }^{2}$ Errui Ding, \\ Ajin Meng, ${ }^{1}$ Liusheng Huang ${ }^{1}$ \\ ${ }^{1}$ University of Science and Technology of China \\ ${ }^{2}$ Department of Computer Vision Technology (VIS), Baidu Inc., China \\ *Corresponding Author. E-mail: qubit@ustc.edu.cn
}

\begin{abstract}
3D object detection is an essential task in autonomous driving and robotics. Though great progress has been made, challenges remain in estimating $3 \mathrm{D}$ pose for distant and occluded objects. In this paper, we present a novel framework named ZoomNet for stereo imagery-based 3D detection. The pipeline of ZoomNet begins with an ordinary 2D object detection model which is used to obtain pairs of leftright bounding boxes. To further exploit the abundant texture cues in rgb images for more accurate disparity estimation, we introduce a conceptually straight-forward module - adaptive zooming, which simultaneously resizes $2 \mathrm{D}$ instance bounding boxes to a unified resolution and adjusts the camera intrinsic parameters accordingly. In this way, we are able to estimate higher-quality disparity maps from the resized box images then construct dense point clouds for both nearby and distant objects. Moreover, we introduce to learn part locations as complementary features to improve the resistance against occlusion and put forward the 3D fitting score to better estimate the 3D detection quality. Extensive experiments on the popular KITTI 3D detection dataset indicate ZoomNet surpasses all previous state-of-the-art methods by large margins (improved by $9.4 \%$ on $\mathrm{AP}_{\mathrm{bv}}(\mathrm{IoU}=0.7)$ over pseudo-LiDAR). Ablation study also demonstrates that our adaptive zooming strategy brings an improvement of over $10 \%$ on $\mathrm{AP}_{3 \mathrm{~d}}(\mathrm{IoU}=0.7)$. In addition, since the official KITTI benchmark lacks fine-grained annotations like pixel-wise part locations, we also present our KFG dataset by augmenting KITTI with detailed instance-wise annotations including pixel-wise part location, pixel-wise disparity, etc.. Both the KFG dataset and our codes will be publicly available at https://github.com/detectRecog/ZoomNet.
\end{abstract}

\section{Introduction}

Accurate 3D object detection is essential and indispensable for many applications, such as robotics and autonomous driving. Currently, leading 3D object detection methods (Yang et al. 2019; Shi et al. 2019) for autonomous driving rely heavily on LiDAR (light detection and ranging) data for acquiring accurate depth information. As LiDAR is known to be expensive and has a relatively short perception range (typically around $100 \mathrm{~m}$ for autonomous driving),

Copyright (c) 2020, Association for the Advancement of Artificial Intelligence (www.aaai.org). All rights reserved.

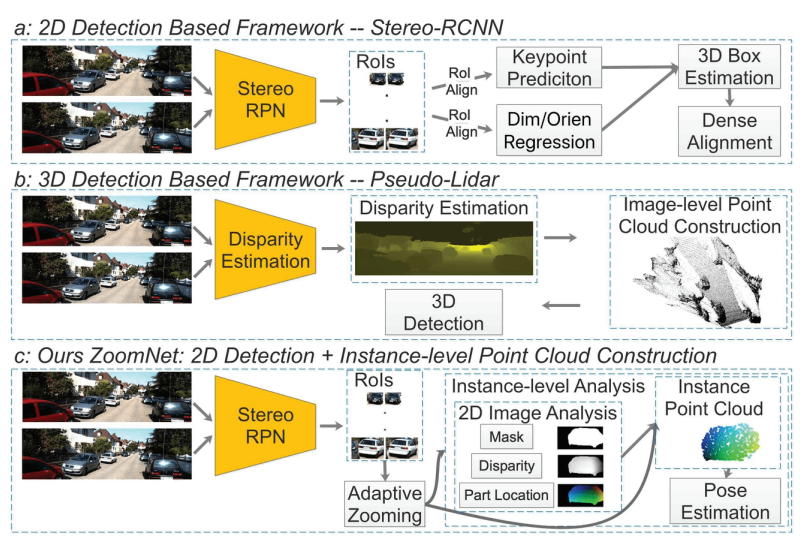

Figure 1: Comparisons between ZoomNet and existing frameworks. Framework (a) performs 2D detection then infer $3 \mathrm{D}$ pose by $3 \mathrm{D}-2 \mathrm{D}$ geometry constraints. Framework (b) estimates the disparity map to construct pseudo LiDAR, then performs 3D detection models on pseudo LiDAR. By contrast, our proposed ZoomNet, as shown in (c), performs instance-level disparity estimation and analysis, yielding superior performance.

stereo cameras which are orders of magnitude cheaper than LiDAR serve as a promising alternative and receive wide attention in both academia and industry. Stereo cameras, which work in a manner similar to human binocular vision, can operate at a higher scanning rate and provide abundant textures cues in high-resolution stereo images for distant objects.

Recent stereo imagery-based 3D detection frameworks put great efforts to explore how stereo 3D information can be effectively used and adapt existing $2 \mathrm{~d} / 3 \mathrm{~d}$ detection models for plugging into their frameworks. As shown in Fig. 1a, recent Stereo-RCNN (Li, Chen, and Shen 2019) first adapts the popular RPN (region proposal networks) for simultaneous $2 \mathrm{~d}$ detection and box association. The concatenated left-right RoI features are used to regress 2D key-points and $3 \mathrm{D}$ dimensions. Then, the $3 \mathrm{D}$ pose is estimated by solving 


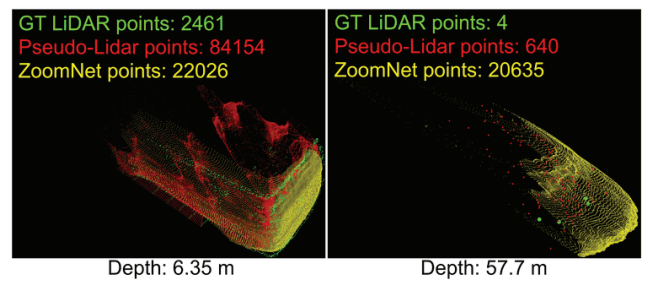

Figure 2: By adaptive zooming, the density of our estimated point cloud is uniform no matter how distant the car is. By comparison, the density of pseudo LiDAR and the GT LiDAR drops dramatically. Moreover, our generated points (in blue) are basically distributed smoothly on the visible surface of the car. Best viewed in color.

3D-2D projections that are constrained by the 3D box corners with corresponding 2D left-right boxes and key-points. However, such geometry constrain-based method usually rely on high quality bounding box and precise key-point localization which are difficult to guarantee due to the commonly occurred occlusions. More recently, as shown in Fig. $1 \mathrm{~b}$, the state-of-the-art framework Pseudo-LiDAR (Wang et al. 2019c) achieved remarkable progress by converting the representation of stereo depth from disparity maps to pseudo-LiDAR point clouds (PCs) and leveraging the 3D detection models. The image-level disparity map is estimated in a holistic manner in Pseudo-LiDAR. However, since the visible pixels are limited by image resolution and occlusions, it is usually hard to exam detailed relative positions between stereo image pair and estimate accurate disparity for distant objects. As a result, Pseudo-LiDAR appears weak performance on distant objects. A comparison between Pseudo-LiDAR and our method is shown in Fig. 5.

Naturally, human's vision system can accurately locate objects in $3 \mathrm{D}$ spaces via attentively watching and analysing each object instance. It can also hallucinate 3D poses for object with severe occlusion based on the prior knowledge of objects' 3D shapes. Such instance-level analysis and shape priors are far from well exploited in existing stereo imagerybased frameworks.

Considering above issues, in this paper, we present a novel framework, as shown in Fig. 1c, named Part-aware Adaptive Zooming neural network (ZoomNet). ZoomNet inherits while extends Pseudo-LiDAR by estimating disparity map and constructing PC for each object instance rather than the full image. To realize high quality instance-level disparity estimation, we propose a novel strategy named adaptive zooming which fully exploits the convenient zoom in/out on stereo images. In adaptive zooming, all RoIs in 2D detection results are adaptively zoomed to the same resolution and camera intrinsic parameters are changed accordingly for projecting pixels on zoomed images to the instance 3D PC. Through adaptive zooming, faraway cars are analyzed at larger resolutions to obtain better disparity estimation and have more compact PCs (see Fig. 2). Moreover, we introduce the pixel-wise part location which indicates the relative $3 \mathrm{D}$ position in the corresponding object for bet- ter 3D detection performance on occlusion cases (see Table 2). Further, several previous 2D detection based methods (Li, Chen, and Shen 2019; Mousavian et al. 2017) utilize 2D classification probability as the 3D detection confidence. We argue that the 3D detection quality, quantified as the 3D IoU between the predicted 3D position and its corresponding ground truth (GT), is not well correlated with the 2D classification probability. Therefore, we put forward the 3D fitting score as a supplementary to the $2 \mathrm{D}$ classification probability for better quality estimation. On the popular KITTI 3D detection benchmark, our ZoomNet surpasses all state-of-theart methods by large margins. Specifically, ZoomNet first reaches a high average precision (AP) of $70 \%$ in the Hard set on $\mathrm{AP}_{3 \mathrm{~d}}(\mathrm{IoU}=0.5)$.

Since the official KITTI 3D detection benchmark only provides the instance $3 \mathrm{D}$ position annotation, we introduce KITTI fine-grained car (KFG) dataset to fill the vacancy of fine-grained annotations and to enable the training of ZoomNet. Due to limited space, we leave the details of KFG in the supplementary material.

We summarize our main contributions as follows:

- We put forward ZoomNet, a novel stereo imagery-based 3D detection framework.

- We present adaptive zooming as an effective strategy by which all instances are analyzed at the same resolution and constructed instance PCs has a uniform density regardless of the instance distance.

- We propose part location to improve the robustness of the network against occlusion and 3D fitting score for better $3 \mathrm{D}$ detection quality estimation.

- Evaluations on KITTI dataset show that ZoomNet outperforms all existing stereo imagery-based methods by large margins. More importantly, it first reaches a high AP of $70 \%$ in the Hard set.

\section{Related Work}

We briefly review two stereo imagery-based 3D object detection frameworks and recently-proposed neural networks related to part locations.

2D detection based. 3DOP (Chen et al. 2017) is a pioneering work on stereo imagery based 3D object detection. It encodes much information into an energy function and use it to generate 3D proposals for 3D location parameter regression. In (Li, Qin, and others 2018), the authors propose a semantic tracking system which converts the complex 3D object detection problem into 2D detection, view-point classification, and straightforward closed-form calculation. Triangulation Learning Network (Qin, Wang, and Lu 2019) performs 3D detection by exploiting 3D anchors to construct instance correspondences between stereo images. Recently, $\mathrm{Li}$, Chen, and Shen extends Faster-RCNN to simultaneously detect and associate objects in stereo images and utilizes the key-points and 2D constraints to estimate an initial 3D position. After that, the dense alignment strategy is applied to enhance depth estimation significantly. However, dense alignment relies on regions between two boundary key-points. When the lower part of an instance is occluded, this instance 


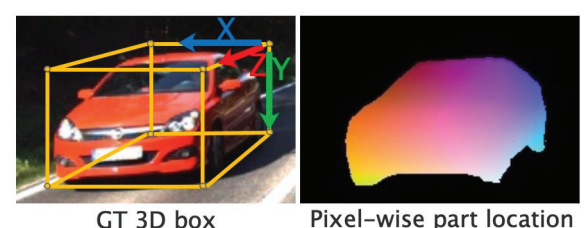

GT 3D box

Figure 3: Illustration of the formulation of part locations. We regard the pixel-wise part location as RGB channels for visualization.

will be discarded. By comparison, our proposed part locations are more robust to occlusion than key-points.

3D detection based. With the rapid development of $\mathrm{Li}$ DAR processing techniques which handle LiDAR data directly, Wang et al. propose the pseudo LiDAR representation obtained by computing dense disparity map and then converting them to pseudo LiDAR representations mimicking LiDAR signal. Recently many monocular image-based methods (Ma et al. 2019; Manhardt, Kehl, and Gaidon 2019; Weng and Kitani 2019) also adopt this representation to achieve high 3D detection performance. After that, the best existing LiDAR-based 3D detection algorithm is adopted in order to detect cars. Converting 2D image pixels to 3D PCs can ease the severe occlusion in 2D images and thus makes detection much easier. Therefore, to date, 3D detection based methods (Wang et al. 2019c; You et al. 2019) achieve higher performances. However, as the depth is calculated at the image level, the estimated depth of distant cars are error-prone. In this paper, we propose adaptive zooming as an effective strategy to obtain better 3D detection results for distant cars.

Part-aware neural networks. As cars' body is rigid, intro-object part locations are informative representations for estimating the pose of cars. Different from key-points, every visible pixel of a car is a valid part location. Recently, in a LiDAR-based method (Shi et al. 2019), the authors also utilize part annotations to help optimize the 3D detection result. However, part locations are still never explored in image-based methods.

\section{Methodology}

As shown in Fig. 4, our proposed ZoomNet contains two major stages: (A) 2D detection and adaptive zooming. (B) instance-wise fine-grained analysis. In stage (A), we perform a joint $2 \mathrm{D}$ object detection and bounding box association on the input stereo images. For each instance detected, a 2D classification probability and sizes of 3D bounding box (or dimension $M$ ) are estimated. In stage (B), ZoomNet performs a fine-grained analysis for all instances, where pixelwise estimations are predicted, including disparity, part location, foreground/background (f/g) labeling. Then, instancewise PCs are constructed for pose estimation utilizing these pixel-wise estimations. In pose estimation, the rotation $R$, translation $T$, and a 3D fitting score measuring the goodness of a 3D bounding box are predicted. Hence, the final output of our ZoomNet for a instance is $(M, R, T, S)$, where the 3D detection confidence $S$ is the product of the 2D classification probability and the 3D fitting score.

\section{D Detection and Adaptive Zooming}

In this stage, given a paired left (denoted as $\mathcal{L}$ ) and right (denoted as $\mathcal{R}$ ) images, the object detector predicts a list of stereo instances and their corresponding dimensions $M$ and 2D classification probability $P$. Then, by adaptive zooming, faraway cars are analyzed on larger resolutions for better depth estimation.

2D Detection. Different from common 2D detection tasks, object detection on stereo images needs to associate region proposals in stereo images. Recently, Stereo-RCNN (Li, Chen, and Shen 2019) extends Faster-RCNN for stereo inputs to simultaneously detect and associate instance in stereo images. Then, the stereo RoI features are extracted and are concatenated to classify object categories and to regress dimensions, orientations, as well as key-points. We adopt Stereo-RCNN as the backbone for 2D detection and abandon the branches for key-points and orientations. An instance RoI containing a pair of stereo boxes can be represented by $(x, \bar{x}, y, w, h)$ where $(x, y, w, h)$ denotes the left RoI $L((x, y), w, h$ is the left-up corner coordinate, the width, and the height respectively) and $(\bar{x}, y, w, h)$ denotes the corresponding right $\mathrm{RoI} R$.

Adaptive Zooming. To obtain better depth estimation for distant cars, we introduce an effective strategy named adaptive zooming (AZ). When we sample pixels from film coordinates (projected image), pixels may be rectangular instead of square (Forsyth and Ponce 2002). Therefore, the size of the resulting pixel array can vary with two zooming parameters: the horizontal zooming parameter $k$ and the vertical zooming parameter $m$. Following the official KITTI notations (Geiger et al. 2013), we denote the left camera and right camera as $P_{2}$ and $P_{3}$ respectively. By choosing different zooming parameters to re-sample pixels, the intrinsics of $P_{2}$ change as follows:

$$
\hat{P}_{2}=\left[\begin{array}{ccc}
k & 0 & 0 \\
0 & m & 0 \\
0 & 0 & 1
\end{array}\right] * P_{2}=\left[\begin{array}{cccc}
k f_{u}^{(2)} & 0 & k c_{u}^{(2)} & -k f_{u}^{(2)} b_{x}^{(2)} \\
0 & m f_{v}^{(2)} & m c_{v}^{(2)} & 0 \\
0 & 0 & 1 & 0
\end{array}\right]
$$

where the superscript (2) indicates it belongs to $P_{2} . f_{u}, f_{v}$ represents the focal length and $c_{u}, c_{v}$ denotes the principal point offset. Besides, $b_{x}$ denotes the baseline with respect to the reference camera. The intrinsic parameters of the right camera $P_{3}$ change to $\hat{P}_{3}$ in the same way. $k$ and $m$ are zooming parameters adjusting the focal length of $P_{2}$ and $P_{3}$. Besides, the zooming parameters also adjust the size of the left RoI $L$ and right RoI $R$ such that the size of resulting images equals to the expected size in the second stage, that is: $k=\mathcal{W} / w, m=\mathcal{H} / h$, where $(\mathcal{W}, \mathcal{H})$ is the pre-defined input image size in stage (B).

Different from the depth estimation in Pseudo-LiDAR based methods (Wang et al. 2019c; You et al. 2019), AZ 


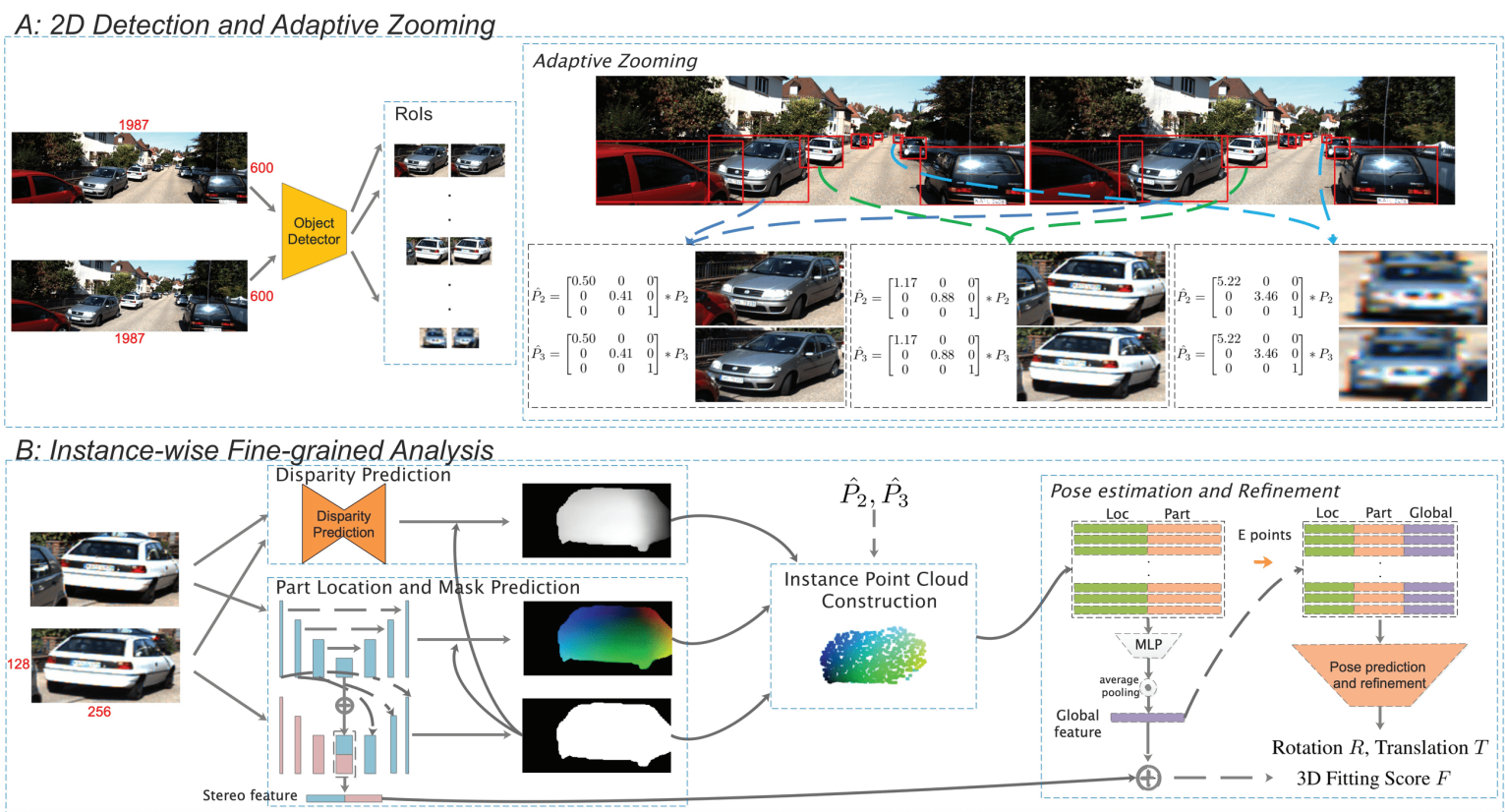

Figure 4: Overview of our proposed ZoomNet. In stage (A), ZoomNet first detects and associates instances from stereo images. Then, it scales all instances to the same dimension and adjusts the camera intrinsics. In stage (B), the disparity map, part location map as well as the segmentation map are jointly estimated. Then, these $2 \mathrm{D}$ feature maps are converted to 3D instance-wise point clouds for subsequent pose estimation and 3D fitting score regression. Best viewed in color.

can reduce the error of depth estimation by $k$ for distant cars by analyzing the disparity on a larger image. Suppose the image is zoomed horizontally by $k$, for a given error in disparity $\delta D, \mathrm{AZ}$ influences the error in depth $\delta Z$ as follows:

$$
Z \propto \frac{k}{D} \Rightarrow \delta Z \propto \frac{k}{D^{2}} \delta D \Rightarrow \delta Z \propto \frac{1}{k} Z^{2} \delta D
$$

The effectiveness of AZ for depth estimation is also demonstrated by comparative experiments on cars at different distances (see Fig. 5).

\section{Instance-wise Fine-grained Analysis}

In this stage, ZoomNet performs fine-grained analysis for all instances. We denote the zoomed left RoI and zoomed right RoI as $\hat{L}$ and $\hat{R}$ respectively. For a left input $\hat{L}$, three pixel-wise predictions are generated, i.e., a disparity map, a part location map, and a f/g labeling. Then, a 3D pose is estimated based on these three predictions. Finally, the goodness of a 3D bounding box estimated is scored by considering both the $2 \mathrm{D}$ classification probability and our proposed 3D fitting score. Thanks to the versatile and flexible deep neural networks, we design an end-to-end trainable networks to accomplish all process, which consists of three subnetworks.

Disparity Prediction Following Pseudo-LiDAR, we adopt PSMnet (Chang and Chen 2018) to perform stereo matching between $\hat{L}$ and $\hat{R}$ with a smaller maximum disparity value because $\hat{L}$ and $\hat{R}$ are roughly aligned.
Different from Pseudo-LiDAR which fixes the network parameters after training, gradients are permitted to proceed to enable end-to-end learning in out ZoomNet. We think that end-to-end learning allows the sub-network to be trained with additional supervisions from subsequent 3D rotation and translation regression, enabling the network to predict disparities more accurately.

Part Location and Mask Prediction We formulate the part label of a foreground pixel as three continuous values $\left(p_{x}, p_{y}, p_{z}\right) \in[0,1]$ to indicate its relative $3 \mathrm{D}$ position in object coordinate. Different from (Shi et al. 2019), our part locations are predicted on 2D pixels rather than 3D points. As shown in Fig. 3, we visualize the part locations by regarding $\left(p_{x}, p_{y}, p_{z}\right)$ as three RGB channels.

We design an encoder-decoder architecture for estimating part location and f/g segmentation mask on $L$ (see Fig. 4). The encoder is shared, but we use different decoders for part locations and b/f segmentation mask. As predicting part locations on $L$ is straightforward, it is ambiguous to decide the interested instance purely on $L$ when two cars overlap heavily. Thus, as shown in Fig. 4, we aggregate the features of $L$ and the features of $R$ extracted by the same encoder in mask prediction to alleviate this ambiguity.

Point Cloud Construction Given the segmentation mask, the part location map, and the left disparity map, we construct PCs from foreground pixels. Converting a pixel in the image plane to a $3 \mathrm{D}$ point in the camera coordinate system needs the pixel coordinate on the $2 \mathrm{D}$ image as well as the 
corresponding camera intrinsic parameters. A common practice is reshaping these 2D feature maps of the height $k w$ and the width $m h$ back to the original width $w$ and height $h$, then use the original camera intrinsic parameters $P_{2}$ and $P_{3}$ to project pixels in the original input image $\mathcal{L}$ to $3 \mathrm{D}$ points. With the help of AZ, we do not need to down-sample or upsample 2D feature maps. Instead, we perform this projection on the hallucinated zoomed left image $\hat{\mathcal{L}}$ using the corresponding pseudo camera intrinsic parameters $\hat{P}_{2}$ and $\hat{P}_{3} \cdot \hat{\mathcal{L}}$ is obtained by scaling $\mathcal{L}$ by $k$ in the horizontal direction and by $m$ in the vertical direction. In this way, the starting point of $L$ changes from $(x, y)$ to $(k x, m y)$ and the disparity offset between $L$ and $R$ changes to $\hat{O}=k(x-\bar{x})$. However, the baseline distance $\hat{b_{l}}$ between $\hat{P}_{2}$ and $\hat{P}_{3}$ stays unchanged:

$$
\begin{array}{r}
\hat{b}_{l}=\left(-k f_{u}^{(2)} b_{x}^{(2)}+k f_{u}^{(3)} b_{x}^{(3)}\right) /\left(k f_{u}^{(2)}\right) \\
=\left(-f_{u}^{(2)} b_{x}^{(2)}+f_{u}^{(3)} b_{x}^{(3)}\right) /\left(f_{u}^{(2)}\right)
\end{array}
$$

For each foreground point $(u, v), u \in[0, \mathcal{W}), v \in[0, \mathcal{H})$ on the segmentation map, we denote the predicted disparity of this point as $d_{u, v}$. The position of a 3D point in camera coordinate, $(x, y, z)$ can be computed as:

$$
\left\{\begin{array}{l}
x=\left(u+k x-k c_{u}^{(2)}\right) * z /\left(k f_{u}^{(2)}\right)+b_{x}^{(2)} \\
y=\left(v+m y-m c_{v}^{(2)}\right) * z /\left(m f_{v}^{(2)}\right) \\
z=\left(k f_{u}^{(2)} \hat{b_{l}}\right) /\left(d_{u, v}+\hat{O}\right)
\end{array}\right.
$$

Also, for each 3D point $(x, y, z)$ in camera coordinate, the part location $\left(p_{x}, p_{y}, p_{z}\right)$ in object coordinate is also integrated. Suppose there are $N$ foreground pixels on the segmentation map, then the dimension of the constructed PC is $N * 6$, where $N * 3$ is the $3 \mathrm{D}$ location and another $N * 3$ is the corresponding estimated part location.

Thanks to adaptive zooming, the constructed instance PC usually has a uniform density (see Fig. 2) no matter the distance of the car is near or far. In ablation study (see Table 4), we will show that the high density PC is beneficial for high quality $3 \mathrm{D}$ detection.

Pose Estimation and Refinement After the instance-wise PC is constructed, we sample a pre-defined number $E$ of points for 3D feature extraction. As points generated by ZoomNet are basically evenly distributed on the instance surface as discussed before, we simply sample $E$ points randomly. The common practice is to estimate the rotation $R$ and the translation $T$ by a direct regression or a combination of classification and regression (Shin, Kwon, and Tomizuka 2018). However, inspired by a recent work named DenseFusion (Wang et al. 2019a) on RGB-D pose estimation, we think that a CAD model-based distance loss can better measure the quality of pose estimation. We hence modify DenseFusion for our pose estimation and tailor it by removing its CNN module and exploiting predicted part locations as the color embeddings.

As aforementioned, the 2D classification probability $P$ is not well correlated with the $3 \mathrm{D}$ detection quality. Therefore, we propose the 3D fitting score to better measure the quality of $3 \mathrm{D}$ detection. However, predicting the quality purely on the constructed PC is very ambiguous. Therefore, we introduce the stereo imagery features because the stereo images features provide many clues related to the $3 \mathrm{D}$ detection quality like the distance, the occlusion level, etc. The stereo imagery features and the global PC features are aggregated and are feed to a fully connected layer for $3 \mathrm{D}$ fitting score regression. The training objective $\hat{F}$ is associated with the mean distance $\hat{D}$ between the constructed instance PC and the GT instance PC constructed by the GT disparity. For each foreground point (take $n$ for example), let $\left[X_{n}, Y_{n}, Z_{n}\right]$ be the generated point coordinate and $\left[\hat{X}_{n}, \hat{Y}_{n}, \hat{Z}_{n}\right]$ be the GT point coordinate. The training target is formulated as:

$$
\begin{aligned}
& \hat{D}=\operatorname{Clip}\left(\frac{1}{E} \sum_{n}\left|Z_{n}-\hat{Z}_{n}\right|\right) \in[0.0,1.0] \\
& \hat{F}=1.0-e^{-\frac{\hat{D}}{\theta}}
\end{aligned}
$$

We clip $\hat{D}$ to the range $[0.0,1.0]$ as the $3 \mathrm{D}$ detection result often fails when the depth error exceeds 1 meter. Since it is hard to estimate depth correctly, we specifically design the loss to enforce the network to focus on depth prediction by using Eq. (5).

\section{Training}

We leave the training details including stereo-RCNN and pose estimation in the supplementary material. In stage (B), the input image size $(\mathcal{W}, \mathcal{H})$ is set to $(256,128)$. $E$ is set to 500 in both training and testing phases because empirically we find sampling more points does not enhance the pose estimation performance. The multi-task loss in stage (B) is formulated as follows:

$$
L=L_{m}+L_{d}+L_{p a}+L_{p o}+L_{r}+L_{s}
$$

where $L_{m}$ is the binary cross-entropy loss between the estimated pixel-wise segmentation score and the GT b/f segmentation mask. For each GT foreground pixel, $L_{d}$ is the smooth L1 distance between the estimated disparity and the GT disparity, and $L_{p a}$ is the smooth L1 distance between the estimated part location and the GT part location on the GT foreground area. $L_{p o}$ and $L_{r}$ are CAD model-based pose estimation loss and refinement loss (refer to (Wang et al. 2019a) for more details), respectively. $L_{s}$ is the smooth L1 distance between the predicted 3D fitting score $F$ and $\hat{F}$. Empirically we find the 3D fitting score cooperates with the $2 \mathrm{D}$ classification probability better when $\theta$ is set to 8 . Moreover, to stabilize the joint training, a lower weight $w=0.1$ is assigned to the loss of part location and mask. The learning rate in pose estimation and refinement is set to 0.1 of the global learning rate.

During training in stage (B), we perform data augmentation by randomly but "moderately" shifting the horizontal starting point of the left/right crops. By default, the left/right crops are cropped at the left/right bounding box of the $2 \mathrm{D}$ detection result. When the horizontal starting points $\bar{x}, x$ of the left/right bounding box change, the pixelwise disparity between the Left/Right crop changes accordingly."Moderately" shifting means that the resulting disparity map should satisfy two requirements: (1) the minimal disparity of foreground pixels should be greater than 
zero; (2) the maximum disparity of foreground pixels should be smaller than the default maximum disparity for stereo matching.

\section{Experiments}

We evaluate our method on the challenging KITTI object detection benchmark (Geiger et al. 2013). The main results are shown in Table 1, where we compare ZoomNet with previous state-of-the-arts. To demonstrate the effectiveness of adaptive zooming, we carry out comparative experiments on cars at different distances. Moreover, We test ZoomNet on different occlusion levels to examine its resistance against occlusion. Lastly, we perform ablation study to provide a deeper understanding of ZoomNet. More details of training ZoomNet can be found in the supplementary material.

\section{Metric}

We focus on $3 \mathrm{D}$ object detection $\left(\mathrm{AP}_{3 \mathrm{~d}}\right)$ and bird's-eyeview $(\mathrm{BEV})$ object detection $\left(\mathrm{AP}_{\mathrm{bv}}\right)$ and report the results on the validation set. $\mathrm{AP}_{3 \mathrm{~d}}$ and $\mathrm{AP}_{\mathrm{bv}}$ are the average precision measured in $3 \mathrm{D}$ and $\mathrm{BEV}$, respectively. Following previous works (Wang et al. 2019c; Li, Chen, and Shen 2019; Chen et al. 2017), we focus on the 'car' category and split the 7481 training images into training set and validation set with roughly the same amount. It is worth noting that all cars are divided into three difficulties: Easy, Mode (moderate) and Hard according to different bounding box heights and different occlusion/truncation levels. Moreover, there is a category named 'DontCare' which denotes regions where objects have not been labeled, and detection results on these regions will be ignored. Therefore, when we compare methods on a specific condition, by default, we set the category of all cars that do not meet this condition as 'DontCare'.

\section{D and BEV object detection}

We compare recent works on stereo imagery-based 3D detection: 3DOP (Chen et al. 2017), MLF (Xu and Chen 2018), DirectShape (Wang et al. 2019b), TLN (Qin, Wang, and Lu 2019), Stereo R-CNN (Li, Chen, and Shen 2019), and Pseudo-LiDAR (Wang et al. 2019c). Except for PseudoLiDAR, all methods are based on 2D detection. PseudoLiDAR provides multiple 3D object detectors for chosen. We select the one with the highest performance. The recently improved version of Pseudo-LiDAR (You et al. 2019) is not included because its code is not publicly available yet.

We summarize the main results in Table 1, where our method outperforms all state-of-the-art methods, especially on the $\mathrm{AP}_{\mathrm{bv}}$ at the IoU thresh of 0.7. Quantitative results are shown in Fig. 6. While adopting the same detector, our method outperforms Stereo R-CNN by large margins on both $\mathrm{AP}_{\mathrm{bv}}$ and $\mathrm{AP}_{3 \mathrm{~d}}$. On the most challenging metric $\mathrm{AP}_{3 \mathrm{~d}}$ (IoU=0.7), we steadily surpass Stereo R-CNN by over $14 \%$ in the Mode and Hard set. Though we only surpass PseudoLiDAR by a small margin on $\mathrm{AP}_{3 \mathrm{~d}}(\mathrm{IoU}=0.7)$ in the Easy set, we report a large improvement of over $9 \%$ on $\mathrm{AP}_{3 \mathrm{~d}}$ $(\mathrm{IoU}=0.5)$ in the most difficult Hard set. More importantly, in the Hard set of $\mathrm{AP}_{3 \mathrm{~d}}(\mathrm{IoU}=0.5)$, our method achieves a high AP of $70.47 \%$. While LiDAR-based detectors do not

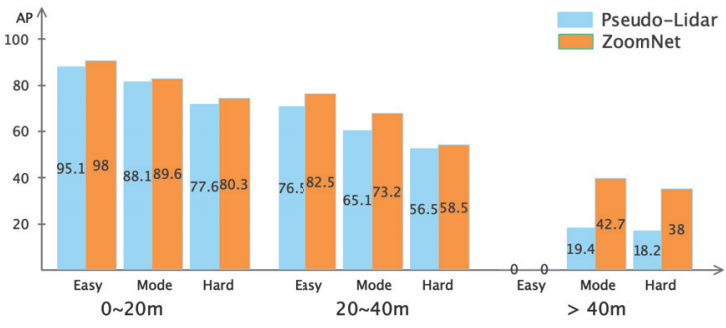

Figure 5: Comparisons between ZoomNet and PseudoLiDAR at different distances on $\mathrm{AP}_{3 \mathrm{~d}}(\mathrm{IoU}=0.5)$.

have the problem of accurate depth estimation, this is the first time that stereo imagery based method achieves over $70 \% \mathrm{AP}$ in the challenging Hard set on $\mathrm{AP}_{3 \mathrm{~d}}(\mathrm{IoU}=0.5)$.

How can ZoomNet achieve a nearly $70 \%$ AP in the challenging Hard set? Since cars in the Hard set are mostly distant and are difficult to see, the reasons may be two-fold: (i) Thanks to adaptive zooming, ZoomNet can detect distant cars more accurately by introducing a distance-invariant strategy. (ii) ZoomNet has strong robustness to occlusion. In the following subsections, we investigate the 3D detection performance at different distances and compare ZoomNet with on different occlusion levels.

\section{Comparisons at Different Distances}

Compared with estimating full image disparity, as explained in Eq. (2), AZ can reduce the depth estimation error for distant cars. To test the effect of AZ on $3 \mathrm{D}$ detection performance at different distances, we divide all cars in the validation set into three categories by depth: (i) $0 \sim 20 \mathrm{~m}$, (ii) $20 \sim 40 \mathrm{~m}$, and (iii) $>40 \mathrm{~m}$. The result is shown in Fig. 5 . For near cars $(0 \sim 20 \mathrm{~m})$, the gap between Pseudo-LiDAR and our ZoomNet is small. Their high performance at the near distance also demonstrates that stereo imagery-based methods are promising for low-speed robots because lowspeed robots, like we humans, do not need to care about objects that are too far away.

With the depth increasing to $40 \mathrm{~m}$ and more, the performance gap is growing larger and larger. Especially in the Hard set, for cars of more than 40 meters away, ZoomNet achieves $42.7 \%$ AP in the Mode set, about $120 \%$ higher than Pseudo-LiDAR. This trend validates that ZoomNet has an obviously better 3D detection performance for distant cars.

\section{Comparisons on Different Occlusion Levels}

To validate that ZoomNet is more robust to occlusion than key-points based methods, we compare ZoomNet with Stereo R-CNN on different occlusion levels. The result on $\mathrm{AP}_{3 \mathrm{~d}}(\mathrm{IoU}=0.7)$ is shown in Table 2. Occlusion Level 0,1,2 represent 'Fully visible', 'Partly occluded', 'Difficult to see', respectively. Here we only care about the comparison in the Hard set because cars belonging to occlusion Level 2 only exist in the Hard set. On all occlusion levels, our ZoomNet steadily surpasses Stereo R-CNN by large margins. Especially on the occlusion level 1 , the performance of ZoomNet 


\begin{tabular}{|c|c|c|c|c|c|c|c|c|c|c|c|c|c|}
\hline \multirow[b]{2}{*}{ Method } & \multirow[b]{2}{*}{ Detector } & \multicolumn{3}{|c|}{$\mathrm{AP}_{\mathrm{bv}}(\mathrm{IoU}=0.5)$} & \multicolumn{3}{|c|}{$\mathrm{AP}_{\mathrm{bv}}(\mathrm{IoU}=0.7)$} & \multicolumn{3}{|c|}{$\mathrm{AP}_{3 \mathrm{~d}}(\mathrm{IoU}=0.5)$} & \multicolumn{3}{|c|}{$\mathrm{AP}_{3 \mathrm{~d}}(\mathrm{IoU}=0.7)$} \\
\hline & & Easy & Mode & Hard & Easy & Mode & Hard & Easy & Mode & Hard & Easy & Mode & Hard \\
\hline $3 \mathrm{DOP}$ & $2 \mathrm{D}$ & 55.04 & 41.25 & 34.55 & 12.63 & 9.49 & 7.59 & 46.04 & 34.63 & 30.09 & 6.55 & 5.07 & 4.10 \\
\hline DirectShape & $2 \mathrm{D}$ & 54.35 & 38.15 & 32.03 & 15.55 & 10.77 & 9.22 & 47.62 & 31.01 & 28.26 & 8.81 & 5.56 & 5.37 \\
\hline MLF & $2 \mathrm{D}$ & - & 53.56 & - & - & 19.54 & - & - & 47.42 & - & - & 9.80 & - \\
\hline TLN & $2 \mathrm{D}$ & 62.46 & 45.99 & 41.92 & 29.22 & 21.88 & 18.83 & 59.51 & 43.71 & 37.99 & 18.15 & 14.26 & 13.72 \\
\hline Stereo-RCNN & $2 \mathrm{D}$ & 87.13 & 74.11 & 58.93 & 68.50 & 48.30 & 41.47 & 85.84 & 66.28 & 57.24 & 54.11 & 36.69 & 31.07 \\
\hline Pseudo-LiDAR & $3 \mathrm{D}$ & 89.00 & 77.50 & 68.70 & 74.90 & 56.80 & 49.00 & 88.50 & 76.40 & 61.20 & 61.90 & 45.30 & 39.00 \\
\hline ZoomNet & $2 \mathrm{D}$ & 90.62 & 88.40 & 71.44 & 78.68 & 66.19 & 57.60 & 90.44 & 79.82 & 70.47 & 62.96 & 50.47 & 43.63 \\
\hline
\end{tabular}

Table 1: Average precision of bird's-eye-view $\left(\mathrm{AP}_{\mathrm{bv}}\right)$ and $3 \mathrm{D}$ boxes $\left(\mathrm{AP}_{3 \mathrm{~d}}\right)$ comparison, evaluated on the KITTI validation set.

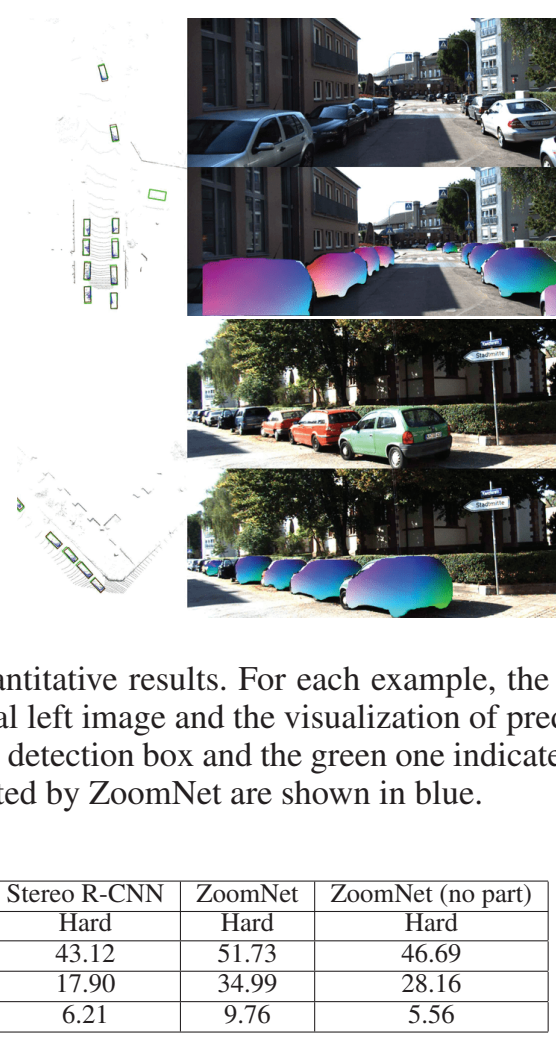

Table 2: Comparisons on different levels of occlusion on $\mathrm{AP}_{3 \mathrm{~d}}(\mathrm{IoU}=0.7)$.

is $95 \%$ higher than Stereo R-CNN. The performance increment demonstrates that ZoomNet performs better than keypoints based methods in occlusion cases. However, when the part location is removed in ZoomNet, the performance on the occlusion level 2 drops a lot and becomes slightly worse than Stereo R-CNN. The tremendous decrease in AP indicates that part locations are crucial for detecting occluded cars. The importance of part locations is also demonstrated in the following ablation study.

\section{Testset Results}

To demonstrate the effectiveness of the proposed model, we also report the evaluation results on the official KITTI test-

\begin{tabular}{|c|ccc|ccc|}
\hline \multirow{2}{*}{ Method } & \multicolumn{3}{|c|}{$\mathrm{AP}_{\text {bv }}(\mathrm{IoU}=0.7)$} & \multicolumn{3}{c|}{$\mathrm{AP}_{3 \mathrm{~d}}(\mathrm{IoU}=0.7)$} \\
\cline { 2 - 7 } & Easy & Mode & Hard & Easy & Mode & Hard \\
\hline Stereo-RCNN & 61.92 & 41.31 & 33.42 & 47.58 & 30.23 & 23.72 \\
\hline Pseudo-LiDAR & 67.30 & 45.00 & 38.40 & 54.53 & 34.05 & 28.25 \\
\hline ZoomNet & $\mathbf{7 2 . 9 4}$ & $\mathbf{5 4 . 9 1}$ & $\mathbf{4 4 . 1 4}$ & $\mathbf{5 5 . 9 8}$ & $\mathbf{3 8 . 6 4}$ & $\mathbf{3 0 . 9 7}$ \\
\hline
\end{tabular}

Table 3: 3D detection and localization AP on the KITTI test set.

ing set in Table 3. The detailed performance can be found online. ${ }^{1}$ It is worth noting that our ZoomNet has surpassed state-of-the-art methods on $\mathrm{AP}_{\mathrm{bv}}(\mathrm{IoU}=0.7)$ by $9.9 \%$ in the Mode set.

\section{Ablation Study}

In this ablation study, we validate the contributions of part locations, 3D fitting score (3DFS), and AZ Results are shown in Table 4 . When not using part locations, we adjust the input channels of pose estimation. When not using

\footnotetext{
${ }^{1}$ http://www.cvlibs.net/datasets/kitti/eval_object_detail.php?

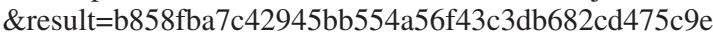




\begin{tabular}{|c|c|c|c|c|c|c|c|c|}
\hline \multirow[b]{2}{*}{ Part } & \multirow[b]{2}{*}{ 3DFS } & \multirow[b]{2}{*}{$\mathrm{AZ}$} & \multicolumn{3}{|c|}{$\mathrm{AP}_{3 \mathrm{~d}}(\mathrm{IoU}=0.5)$} & \multicolumn{3}{|c|}{$\mathrm{AP}_{3 \mathrm{~d}}(\mathrm{IoU}=0.7)$} \\
\hline & & & Easy & Mode & Hard & Easy & Mode & Hard \\
\hline- & - & - & 85.49 & 74.61 & 65.41 & 45.80 & 38.33 & 33.01 \\
\hline- & $\sqrt{ }$ & $\sqrt{ }$ & 88.54 & 77.99 & 68.20 & 54.22 & 40.87 & 34.51 \\
\hline$\sqrt{ }$ & & $\sqrt{ }$ & 88.55 & 77.64 & 68.81 & 57.51 & 46.67 & 40.88 \\
\hline$\sqrt{ }$ & $\sqrt{ }$ & - & 87.00 & 76.51 & 67.36 & 52.07 & 40.39 & 34.20 \\
\hline$\sqrt{ }$ & $\sqrt{ }$ & $\sqrt{ }$ & 90.44 & 79.82 & 70.47 & 62.96 & 50.47 & 43.63 \\
\hline
\end{tabular}

Table 4: Ablation study on ZoomNet. 3DFS denotes 3D fitting score.

3DFS, we regard the $2 \mathrm{D}$ classification probability as the $3 \mathrm{D}$ detection confidence. When not using AZ, we reshape 2D feature maps back to the original size and project pixels in $\mathcal{L}$ rather than on the zoomed image $\hat{\mathcal{L}}$ to construct PCs.

On $\mathrm{AP}_{3 \mathrm{~d}}(\mathrm{IoU}=0.5)$, the absence of part location or $3 \mathrm{DFS}$ leads to a small performance drop of $2 \%$ in the Mode set. However, at the higher 3D IoU threshold 0.7 , non-trivial drops of $10 \%$ and $4 \%$ are observed when part location or $3 \mathrm{DFS}$ is removed. The small gap on $\mathrm{AP}_{3 \mathrm{~d}}(\mathrm{IoU}=0.5)$ and the relatively large gap on $\mathrm{AP}_{3 \mathrm{~d}}(\mathrm{IoU}=0.7)$ demonstrate that part location and 3DFS are crucial for high-quality 3D detection.

When AZ is removed, the PC density decreases dramatically for distant cars. For cars which are 55 meters away, the number of points drops from $20 \mathrm{k}$ to less than $1 \mathrm{k}$. The performance also drops dramatically. In practice, we observe that the end-to-end training in stage (B) also becomes unstable.

Combining these three ingredients together, large improvements on $\mathrm{AP}_{3 \mathrm{~d}}$ (IoU=0.7) are observed, and our integrated ZoomNet pipeline achieves the best 3D detection results among current stereo-imagery based methods.

\section{Conclusion}

In this paper, we propose a novel framework for stereo imagery-based 3D detection. By exploiting adaptive zooming to process cars at various distances at a unified resolution and utilizing part locations to solve the ambiguity under severe occlusion, our method ZoomNet surpasses all existing image-based methods by large margins on the challenging KITTI 3D and BEV object detection tasks. More importantly, ZoomNet is the first image-based method to achieve a high AP of $70 \%$ in the Hard set. The high 3D detection performance demonstrates that ZoomNet can serve as a competitive and reliable backup for autonomous driving and robot navigation.

\section{Acknowledgment}

This work was supported by the National Natural Science Foundation of China (No. 61602400) and the Anhui Initiative in Quantum Information Technologies (No. AHY150300).

\section{References}

Chang, J.-R., and Chen, Y.-S. 2018. Pyramid stereo matching network. In Proceedings of the IEEE Conference on Computer Vision and Pattern Recognition, 5410-5418.

Chen, X.; Kundu, K.; Zhu, Y.; Ma, H.; Fidler, S.; and Urtasun, R. 2017. 3d object proposals using stereo imagery for accurate object class detection. IEEE transactions on pattern analysis and machine intelligence 40(5):1259-1272.

Forsyth, D. A., and Ponce, J. 2002. Computer vision: a modern approach. Prentice Hall Professional Technical Reference.

Geiger, A.; Lenz, P.; Stiller, C.; and Urtasun, R. 2013. Vision meets robotics: The kitti dataset. The International Journal of Robotics Research 32(11):1231-1237.

Li, P.; Chen, X.; and Shen, S. 2019. Stereo r-cnn based 3d object detection for autonomous driving. In $C V P R$.

Li, P.; Qin, T.; et al. 2018. Stereo vision-based semantic 3d object and ego-motion tracking for autonomous driving. In Proceedings of the European Conference on Computer Vision (ECCV), 646661.

Ma, X.; Wang, Z.; Li, H.; Ouyang, W.; and Zhang, P. 2019. Accurate monocular $3 \mathrm{~d}$ object detection via color-embedded $3 \mathrm{~d}$ reconstruction for autonomous driving. arXiv preprint arXiv:1903.11444.

Manhardt, F.; Kehl, W.; and Gaidon, A. 2019. Roi-10d: Monocular lifting of $2 \mathrm{~d}$ detection to $6 \mathrm{~d}$ pose and metric shape. In Proceedings of the IEEE Conference on Computer Vision and Pattern Recognition, 2069-2078.

Mousavian, A.; Anguelov, D.; Flynn, J.; and Kosecka, J. 2017. 3d bounding box estimation using deep learning and geometry. In Proceedings of the IEEE Conference on Computer Vision and Pattern Recognition, 7074-7082.

Qin, Z.; Wang, J.; and Lu, Y. 2019. Triangulation learning network: from monocular to stereo $3 \mathrm{~d}$ object detection. arXiv preprint arXiv:1906.01193.

Shi, S.; Wang, Z.; Wang, X.; and Li, H. 2019. Part-a^2 net: 3d partaware and aggregation neural network for object detection from point cloud. arXiv preprint arXiv:1907.03670.

Shin, K.; Kwon, Y. P.; and Tomizuka, M. 2018. Roarnet: A robust $3 \mathrm{~d}$ object detection based on region approximation refinement. arXiv preprint arXiv: 1811.03818.

Wang, C.; Xu, D.; Zhu, Y.; Martín-Martín, R.; Lu, C.; Fei-Fei, L.; and Savarese, S. 2019a. Densefusion: $6 \mathrm{~d}$ object pose estimation by iterative dense fusion. In Proceedings of the IEEE Conference on Computer Vision and Pattern Recognition, 3343-3352.

Wang, R.; Yang, N.; Stueckler, J.; and Cremers, D. 2019b. Directshape: Photometric alignment of shape priors for visual vehicle pose and shape estimation. arXiv preprint arXiv:1904.10097.

Wang, Y.; Chao, W.-L.; Garg, D.; Hariharan, B.; Campbell, M.; and Weinberger, K. Q. 2019c. Pseudo-lidar from visual depth estimation: Bridging the gap in $3 \mathrm{~d}$ object detection for autonomous driving. In Proceedings of the IEEE Conference on Computer Vision and Pattern Recognition, 8445-8453.

Weng, X., and Kitani, K. 2019. Monocular 3d object detection with pseudo-lidar point cloud. arXiv preprint arXiv:1903.09847.

Xu, B., and Chen, Z. 2018. Multi-level fusion based 3d object detection from monocular images. In Proceedings of the IEEE Conference on Computer Vision and Pattern Recognition, 2345-2353.

Yang, Z.; Sun, Y.; Liu, S.; Shen, X.; and Jia, J. 2019. Std: Sparse-to-dense $3 \mathrm{~d}$ object detector for point cloud. arXiv preprint arXiv:1907.10471.

You, Y.; Wang, Y.; Chao, W.-L.; Garg, D.; Pleiss, G.; Hariharan, B.; Campbell, M.; and Weinberger, K. Q. 2019. Pseudo-lidar++: Accurate depth for $3 \mathrm{~d}$ object detection in autonomous driving. arXiv preprint arXiv:1906.06310. 